\title{
Editorial
}

\section{Ökologiebewegung und Arbeiterbewegung - ein Widerspruch?}

Zwischen Arbeiterbewegung und Ökologiebewegung (oder ,neuen sozialen Bewegungen" schlech thin) ist ein Widerspruch entstanden. Diesen Eindruck jedenfalls gewinnt man zumindest dann, wenn man Verlautbarungen von manchen Gewerkschaftsvertretern hört oder - von der anderen Seite - Stellungnahmen radikaler Ökologen liest, die die Arbeiterbewegung abgeschrieben haben und auf einen neuen Konservativismus der Naturerhal tung gegenüber den zerstörerischen Tendenzen der industriellen Zivilisation setzen. Der Führer der Radikaien Partei Italiens, Marco Panella, geht hier sogar so weit, jeden Bündnispartner, und sei es den Teufel, gegen die atomare Industrialisierung zu akzeptieren und dabei jegliches Zugeständnis auf anderem Sektor zu machen, sei es im Hinblick auf die Frauenbefreiung, die Abtreibungsfrage, die Probleme der Homosexuellen oder auch ganz traditionell bei den Interessen der Arbeiter an Reproduktion der Arbeitskraft und an einem sicheren Arbeitsplatz. Insbesondere seitdem sich in der Bundesrepublik an der Ökologiefrage politische Organisationen gebildet haben und eine Partei „Die Grünen“ entstanden ist, stellt sich die Frage nach der Bewegung des Widerspruchs zwischen Ökologieund Arbeiterbewegung innerhalb dieser Gesellschaft und ihres politischen Systems.

Vielen Ökologisten und ihren theoretischen Vertretern scheint es, als ob im Widerspruch von Ökologie- und Arbeiterbewegung die Ökologiebewegung die Position des Fortschritts einnehme, während die Arbeiterbewegung mit ihren politischen Konzeptionen, Fragestellungen, Zielsetzungen und Strategien hoffnungslos den eigentlichen Anforderungen der Zeit hinterherhinke. Die Arbeiterbewegung vertrete nur ein borniertes Klasseninteresse, während die Ökologiebewegung sich den Problemen der Gattung Mensch annehmen würde. So sind wir mit einer seltsamen, gar überraschenden Verschiebung konfrontiert: während in den Marx'schen Frühschriften, in denen das Gattungswesen Mensch noch emphatisch hervorgehoben wurde, immer klar war, daß die Befreiung des Menschen nur mit der Befreiung der Arbeiterklasse zusammenfallen kann, und umgekehrt die Befreiung der Arbeiterklasse gleichbedeutend mit der Befreiung der Menschheit von ihren gesellschaftlichen und naturlichen Fesseln ist, werden nun die Interessen der Arbeiterklasse als besondere und nicht mehr allgemeine aufgefaßt, auch wenn sich Ök ologen (noch) auf Marx berufen. Die Interessen an Wachstum und Wohlstandssteigerung führen innerhalb dieses Systems dazu, daß dies auf Kosten der inneren und äußeren Natur des Menschen geschieht und daher eine lebensbedrohliche Spirale der Ausnutzung und Zerstörung der Natur in Gang gesetzt werden kann. Das Allgemeinere und Gattungsgeschichtliche in den Interessen der Ökologiebewegung definiert sich infolgedessen schlicht daraus, daß für die Bedingungen des Überlebens der Menschheit überhaupt gegen die Unzuträglichkeiten der industriellen Zivilisation gekämpft wird. 
So wird zum Ansatzpunkt der Ökologiebewegung das Verhältnis von Natur und Gesellschaft, wobei die Gesellschaft weniger als kapitalistische denn als industrielle Gesellschaft mit spezifischem Wachstumsimpuls interpretiert wird. An diesem Punkt haben wir eine entscheidende Nahtstelle für das Verständnis des Verhältnisses von Arbeiterbewegung und Ökologiebewegung. Denn Gesellschaft als solche besagt zunächst einmal gar nichts, wenn nicht ihre Form berücksichtigt und dies in entsprechende politische Entwürfe eingearbeitet wird. Entscheidend wird dies beim Verständnis der Herrschaftsmechanismen, die es ja gerade zu unterlaufen oder besser zu überwinden gilt, um auf andere Impulse setzen zu können als diejenigen des ,quantitativen Wachstums". Wir möchten behaupten: Sofern Herrschaft als rein bürokratisch-technokratische mißverstanden, der Staat dabei als eine „Megamaschine“ fehlin terpretiert und dabei die ganze Tradition der marx istischen oder auch kritischen Staatstheorie über Bord geworfen wird, kann kein politisches Konzept entstehen, um den Herrschaftsbe dingungen dieser Gesellschaft, auch der destruktiven Beherrschung der Natur, beizukommen. Vor allem ist dabei von Bedeutung, daß3 gesellschaftliche Herrschaft über die Natur, ihre Ausplünderung und die für die menschliche Entwicklung lebensbedrohlichen Rückwirkungen, gerade als ein ökonomischsozialer und politischer Prozeß organisiert sind, wobei die kapitalistische Form dieser Gesellschaft in alle Verästelungen, Abläufe, Regelungen hineinragt. Es ist also nicht nur wichtig, daß überhaupt Einsicht in die Ausmaße der Umweltkrise, den Grad der Zerstörung der inneren und äußeren Natur entsteht - wozu Bücher wie dasjenige von Gruhl einen unschätzbaren Beitrag geleistet haben -, sondern daß die gesellschaftliche Organisation dieser Mißwirtschaft damit in einen Zusammenhang gebracht wird: Der Zweck der Kapitalverwertung, aus Wert mehr Wert zu machen, ordnet sich herrschaftlich sow ohl die Produzenten der Werte im Arbeits- und sogar ihren gesamten Lebensprozeß als auch die natürlichen Ressourcen in einer bestimmten, nicht unbedingt naturadäquaten, in jedem Fall aber kapitaladäquaten Technologie unter. Und die Ausformung des politischen Systems entspricht letztlich dieser Zwecksetzung; diese Herrschaft vermittelt sich durch entsprechende Institutionen und soziale Beziehungen, die zu Herrschaftsblöcken, Machtblöcken zusammengefaßt werden. In den institutionell vermittelten gesellschaftlichen Prozeß der Herrschaftssicherung sind die Organisationen der Arbeiterbewegung teilweise, zeitweise und widersprüchlich einbezogen, aber mit ihren Interessen und Ansprüchen bleiben sie der Herrschaft, ihrem Inhalt, ihrem Zweck grundsätzlich subaltern. So haben wir es mit dem strategischen Problem der subalternen Klassen und Interessen zu tun, einen Machtblock aufzubauen, der die Potenzen des herrschenden Blocks zu unterminieren vermag. Um in der Terminologie der Gramscianisch inspirierten eurokommunistischen Diskussion zu sprechen, würde es darauf ankommen, in den sozialen Auseinandersetzungen, die heute zu einem großen Teil um die Naturerhaltung gegen die zerstörerischen Tendenzen des "industriellen Systems“ geführt werden, die Hegemonie gegenüber den auf Wachstum, d.h. auf Kapitalverwertung um jeden Preis setzenden Interessen zu erobern. So betrach tet erscheint die ge- 
sellschaftliche Vermittlung und die Verwirklichung von gegen Zwecksetzung, Form und deren herrschaftliche Absicherung gerichteten Interessen sowohl der grünen wie der Arbeiterbewegung kompliziert genug, jedenfalls nicht als ein Problem, das auf der Ebene Natur - Menschheitsinteresse gegen den maßlosen Quantitativismus des Industriesystems lösbar wäre.

Wenn wir auf diese sehr verkürzt dargestellte Weise Gesellschaft nicht als bloß rationalisierte, technokratisierte, bürok ratisierte, von der Megamaschine Staat dominierte verstehen, sondem selbst als eine widersprüchliche, durch Herrschaftsp rozesse zusamme ngehal tene und durch Strategien der Arbeiterbewegung und der subalternen Schichten immer wieder in Frage gestellte begreifen, dann eröffnet sich ein Ausblick auf die Komplexität der Verhältnisse, in denen sich die Ökologiebewegung auf „,Gesellschaft" bezieht. Auch wenn es einen je nach den historischen Bedingungen von Land zu Land unterschiedlich breiten Korridor von Kompromissen zwischen ,herrschendem" und ,subalternem" Block gibt, so ist eine Überwindung der das Ökologieproblem zumindest verschärfenden kapitalistischen Herrschaftsformen nur möglich in Einordnung und möglicherweise Veränderung des oppositionellen, politischen und sozialen ,Blocks", innerhalb dessen die Arbeiterklasse die Hegemonie besitzt. (Vgl. dazu den Aufsatz von Adalbert Evers „Die politische Bedeutung der sozialen Bewegungen" in: Österreichische Zeitschrift für Politikwissenschaft, Januar 1980).

Wenn von Hegemonie in diesem Zusamme nhang die Rede ist, dann muß also sogleich hinzugefuigt werden, daß Hegemonie kein Zustand, sondern ein Prozeß ist. Und wenn wir in diesem Zusammenhang heute oft genug geneigt sind, die Ökologiebewegung als die dynamische Kraft, die Arbeiterbewegung als in die Defensive geraten zu begreifen, dann müssen wir uns an die Zeit vor 10 bis 12 Jahren zurückerinnem, wo es gerade die Arbeiterbewegung in Westeuropa gewesen ist, die in der Mairevolte in Frankreich, im heißen Herbst in Italien, in den spontanen Streikbewegungen in Deutschland, in der Shop-Steward-Bewegung in England mit militanten Kämpfen gegen das Kapital angetreten war. Bezugspunkt der mit der „68er-Bewegung“ entstehenden ,Neuen Linken“" war ohne Frage die Arbeiterbewegung, auch wenn die neue Linke von ihrer eigenen sozialen Herkunft her zu einem großen, vielleicht überwiegenden Teil dem intellektuellen Milieu entstammte. Im Vordergrund der Politikinhal te standen demnach Reproduktionsinteressen der Lohnabhängigen: Löhne, Arbeitsbedingungen, Lebensverhältnisse. Die Kämpfe selbst fanden an den zentralen Schauplätzen Belegschaften von Fabriken in Kämpfen um Arbeitsbedingungen und Löhne, Arbeiter aus den Wohnvierteln, wenn es um Probleme des Lebensmilieus in den Städten ging. So war es jedenfalls in Italien und zum Teil auch in Frankreich und nach dem Fall der faschistischen Diktaturen in der ersten Hälfte der 70er Jahre auch in Portugal und Spanien. Heute müssen wir uns fragen, wieso diese A rbeiterbewegung und die neue Linke ofensichtlich nicht mehr die ehemalige Dynamik au fweisen. Die Frage nach dem Verhältnis von Ökologie bewegung und Arbeiterbewegung ist demnach auch die Frage nach den Gründen dafür, warum die Arbeiterbewegung nach dem „Kampfzyklus“ der späten 60er und zumindest der ersten Hälfte der 70er Jahre heute in eine Defensive geraten ist. Erst auf diesem Hinter- 
grund ist es verständlich, daß die, neuen sozialen Bewegungen“" die Bedeutung erlangen konnten, die sie Ende der 70er, Anfang der 80er Jahre haben. Doch reicht es nicht aus, die wachsende Bedeutung der Ökologiebewegung als ,kompensierenden Pendelausschlag" gegen die Schwierigkeiten der Arbeiterbewegung heute zu verstehen. Es kommt noch hinzu, daß tatsächlich das Problem der ökologischen Krise immer mehr und bei immer breiteren Massen zu Bewußtsein gekommen und der Eindruck entstanden ist, dak die Parteien des herrschenden Blocks (d.h. verkürzt gesprochen: das Bonner Parteienikartell) zu einer Lösung der aus der Krise entstandenen Probleme nicht fähig sind und eine neue Kraft sich der Probleme annehmen muß. Nur das Zusammenwirken dieser drei Elemente - die Krise der traditionellen Arbeiterbewegung, das tatsächliche Wachstum der ökologischen Probleme, ihr Be. wußstwerden bei breiten Kreisen der Bevölkerung und schließlich die Einsicht in die Unfähigkeit der Parteien des herrschenden Blocks, die ents tandenen Probleme zu lösen - kann die jüngsten Wahlerfolge der grünen Bewegung erklären, die heute (Ende März 1980) in zwei Landesparlamenten vertreten sind und damit zum ersten Mal seit 1953 eine in vieler Hinsicht linke Alternative auf parlamentarischer Ebene repräsentieren. In diesem Zusammenhang sei nur darauf hingewiesen, daß die Grünen (oder die Ökologiebewegung der 70er Jahre schlech thin) das Umweltproblem nicht entdeckt haben: bereits 1961 führte die SPD ihren Wahlkampf in Nordrhein-Westfalen unter dem Slogan der „Blauen Luft über der Ruhr“" und schließlich ist in dieser Hinsicht auch einiger Erfolg mit der Stillegung vieler Kohlenzechen und der Ansiedlung von Industrien, die die Umwelt weniger belasten, erzielt worden, Trotz kleiner Erfolge, auf die die Bundesregierung mit Stolz verweist, sind die Lebensformen und Reproduktionsbedingungen der Menschen nicht besser, eher schlechter geworden, Dabei spielt die Belastung der äußeren und der inneren Natur mindestens eine ebenso große Rolle wie die kaputt machenden Wirkungen der kapitalistisch kompensierenden Eroberung der menschlichen Bedürfnisse und der Unfähigkeit, die gesellschaftlichen Beziehungen der Menschen untereinander menschlich, emanzipatorisch, befriedigend zu gestalten. Daraus erklärt sich auch, daß die Alternativbewegungen, die Ökologie- und die grüne Bewegung, dort am stärksten sind, wo alternative Kommunikationsstrukturen am „dichtesten" sind: in den städtischen Agglomerationen und dann auch noch dort, wo Sensibilität für diese Probleme quasi institutionalisiert ist: nämlich in Universitätsstädten. Natürlich kennt diese Regel Ausnahmen, aber empirische Evidenz auch bei den jüngsten Landtagswahlen in Baden-Württemberg spricht für sie.

\section{Politikverständnisse der grünen Bewegungen}

Den - vordergründigen - Widerspruch von Arbeiterbewegung und Ökologiebewegung haben wir bislang auf dem Hintergrund der Organisation des gesellschaftlichen Herrschaftsprozesses, der Defensive der Arbeiterbewegung seit Eintritt der Krise Mitte der 70er Jahre und dem Bewußtwerden von Ökologieproblemen bei breiten Bevölkeningsschichten diskutiert. Herrschaft haben wir dabei als gesell- 
schaftlich vermittelte Bildung von Blöcken interpretiert, wobei deren Wirksamkeit durch die Institutionen der Gesellschaft prozeßhaft hindurchgeht. Eine Verengung auf Institutionen des politischen Systems darf hier nicht vorgenommen werden, realisieren sich Herrschaftsbeziehungen im Kapitalismus doch in erster Linie durch die ökonomischen Institutionen - Sicherung der Profitabilität, Akkumula tion von Kapital - und deren widersprüchliche Abläufe. Mit entsprechenden politisch-strategischen Schlußfolgerungen werden aber auch andere Interpretationsmuster von Herrschaft entfaltet, die für die zumindest vordergründige Widersprüchlichkeit von Arbeiter- und ökologiebewegung von Belang sind und - so meinen wir - eine Vermittlung dieses Widerspruchs im Sinne seiner ,synthetischen“ Auflösung unmöglich gestalten.

1. Wie Christel Neusüß in ihrem Beitrag in dieser Ausgabe der ProKla belegt, kritisieren manche Theoretiker der Ökologiebewegung das Überhandnehmen der „Megamaschine" Staat. Tatsächlich ist der Staat zu dieser bürokratisierten Maschinerie geworden, aber nur insofern, als er politisch die Defizienzen zu kompensieren versucht, die gesellschaftich infolge der Form kapitalistischer Akkumulation imme $r$ wieder produziert werden, ohne über die Symptome hinaus die Ursache wirklich in den G riff zu bekommen. In diesem Zusammenhang entsteht ein „Bruch zwischen dem Sozialen und dem Politischen" (Gorz), der immer dann eintritt, wenn die Parteien sich der Wuinsche und Revolten der Massen annehmen, Aber diese Politik ist eindimensional geworden, denn dadurch, daß gerade die Parteien sich der Wünsche und Hoffnungen der Massen annehmen, wird ein Teil der Veränderungsdynamik aus der politischen Sphäre herausgefiltert. Die staatliche Form und nicht mehr die gesellschaftliche F orm der Vergesellschaftung, verbunden mit entsprechender politischer Repression, tre te sie nun in Form der Selek tion zu verarbeitender Interessen oder in deren politischer Repression auf, führt auf der einen Seite zu Vergesellschaftungsdefiziten und $\mathrm{d}$. h. politischer Subaiternität gegenüber staatlicher Gängelung, und auf der anderen Seite zu Bewegungen, die sich dezentral außerhalb der staatlichen Zentralisierungsbemühungen ansiedeln und auf diese Weise dem Sog der „Megamaschine Staat “ zu entgehen trach ten, um ein menschliches, heiles Leben al ternativer Vergesellschaftung zu experimentieren. Die Einschätzung des Staates ist irgendwie traditionell; sie folgt dem Muster, das mit der Bestimmung des Staates als „neben und außerhaib" der Gesellschaft existierend gezeichnet wird. Wenn man die subtiler Ausführungen dazu von Marx und anderen vernachlässigt, dann kann daraus politisch strategisch die Schlußfolgerung abgeleitet werden, daß der Staat von den sozialen Bewegungen und ihren Organisationen in der Gesellschaft zu bekämpfen, zu erobern, zu destruieren sei - wie es zum politischen Programm der Dritten Internationale bis zu ihrer Auflösung in den 40er Jahren geworden ist. Heute wird von den ,neuen sozialen Bewegungen“ nicht mehr der Anspruch erhoben, diesen $S$ taat zu erobern und zu zerstören, um auf seinen Trümmern einen wie auch imme $r$ verstandenen Sozialismus aufzubauen, sondern außerhalb, draußen zu bleiben, sich also den polypenhaften Fangarmen der Megamaschine zu entziehen. Dieser "Movimentismus" der sozialen Bewegungen ist allerdings in gewisser Hinsicht ein abgewandelter „Drittin ternationalismus“ hinsichtlich der Einschätzung der Rol- 
le des Staates und des politischen Systems (vgl. dazu die Beiträge in E. Altvater und O. Kallscheuer, Hrsg., „Den Staat diskutieren“, Berlin-West 1979). Denn zentrale Idee in diesem Politikverständnis ist eine bipolare Struktur von Gesellschaft: Die Herrschaft ist staatlich-institutionell vermittelt und weitgehend geschlossen, während die Politik zur Durchsetzung ihrer Interessen bei den gesellschaftlichen Bewegungen außerhalb der Institutionen bleibt. Wie Gorz es formuliert: Die Politik findet nicht mehr in der Politik statt. Veränderungen des gesellschaftlichen und damit auch des politischen Systems werden durch ,Netzwerke“ alternativer Projekte, durch eine A rt ,revolu tionärer Infrastruk tur" (Theo Pinkus) herbeigeführt, also von außerhalb „der Institutionen“. Wie Evers schreibt: ,Drastisch reduziert werden soll vor allem auch die Rolle und Autorität der politischen Parteien gegenüber den sozialen Bewegungen und selbstverwalterischen Initiativen in der Gesellschaft; als Elemente der Kohäsion und des Konsens übernehmen selbstverwalterisch gesellschaftliche Organe immer mehr Aufgaben, während die Parteien den Anspruch aufgeben müssen, auf alles eine Antwort zu haben, und sich im wesentlichen darauf beschränken sollen, die Debatte über gesellschaftliche Alternativen wachzuhalten, als motorische Elemente auf der Suche nach globalen Lösungen zu fungieren, die den vielfältigen Organen einer erweiterten gesellschaftlichen Selbstverwaltung aus dem Blick geraten könnten." (A. Evers, a.a.O., S. 15)

$\mathrm{Daß}$ in dieser Bestimmung des Verhältnisses von alternativer Bewegung und politischem System eine Verkürzung liegt, zeigt auch die Erfahrung. Immer wenn sich die Alternativbewegung zu größeren Demonstrationen ihrer altemativen Macht zusammenschließt, gerät sie in Konfrontation mit der - polizeilich ausgerüsteten, bewaffneten - Macht der, ,ersten Gesellschaft". Alternative Projekte können also funktionieren, sofern sie politisch kalmiert sind und sich eine ökonomische Basis in den Nischen des kapitalistischen Drachen verschaffen. Sofem sie aber das politische System - von außen - zu verändern trachten, werden sie unmittelbar nicht nur mit der Megamaschine Staat konfrontiert, sondem auch mit den Mechanismen gesellschaftlicher Herrschaft durch Presse, Rundfunk, Fernsehen und die herrschenden Bewußtseinsformen bei den Massen der Bevölkerung, die zum größ ten Teil eben Arbeiter und Angestellte, Lohnabhängige, selbst den kapitalistischen Herrschaftsmechanismen Unterworfene sind.

2. Ist die Macht in diesen geschilderten Vorstellungen noch eindeutig bei der Megamaschine Staat lokalisierbar, so steckt sie in den Vorstellungen der ,neuen Philosophen" diffus überall und in uns. Es gibt also auch keine gesellschaftliche Scheidelinie für Macht und ihre Ausuibung, so daß eine Strategie gegen die Mächtigen und ihre Politik von dieser Vorstellung her nicht mehr ableitbar ist. Folgerichtig geht es auch nicht mehr um ,Makropolitik“, sondem um eine „Mikropolitik“, die sich an den eigenen Wünschen und Bedürfnissen orientiert und auf diese Weise ,,subjektive Verkettungen " (Guattari) generiert. Netzwerke sind auch hier die Folge von sozialer Aktion und bilden zugleich den individuell-sozialen Widerstand gegen die Repression aus der gesamtgesellschaftlichen Struktur heraus. Diese Theorie ist keine Theorie einer sozialen Bewegung, dazu bleibt sie viel zu inhaltsleer und sie kann folglich auch weder für die Ökologiebewegung noch für die traditionelle Arbeiterbewegung 
Wege des politischen Handelns weisen. Die Ausblendung der Organisation der Macht zu Machtblöcken macht diese The orie vergleichsweise perspektivlos, auch wenn sie fantastische Reisen anregt: Tunix macht sich auf zu neuen Ufern, zu einem Land, wo nicht unbedingt die Zitronen blühen, aber wo der Strand nicht zugepflastert ist.

\section{Die Bildung der grünen Partei gegen die Tradition der Sozialdemokra tie}

Unser Kurzrefe rat von drei Theorieansätzen der ,neuen sozialen Bewegungen“ des Gramscianisch-eurokommunistisch inspirierten, des movimentistischen, den Gegensatz von Staat und Gesellschaft hervorhebenden, und des auf die eigenen Wünsche und Bedürfnisse gegen die Diffusion de r Macht setzenden - verweist auf Probleme, vor denen die sozialen Bewegungen heute stehen, wenn wir das Verhältnis zur Arbeiterbewegung und ihren Organisationen reflektieren. Das unseres Erachtens entscheidende Problem besteht darin, daß die Arbeiterbewegung mit ihren Organisationen im Prozeß der „Institutionalisierung des Klassenkonflikts" (Dahrendorf) selbst in den Bereich des organisierten, institutionalisierten politischen Systems und in den staatlichen Apparat eingetreten ist. Man kann auch sagen, die Arbeiterbewegung sei, sofern sie organisiert ist, in tegriert worden. Jedoch vergißt diese kritisch gemünzte Feststellung die Tatsache, daß die Arbeiterbewegung im Rahmen dieses Integrationsprozesses bestimmte Aufgaben der Sicherung der Reproduktion der Lohnarbeit übernimmt, die dem Staat in schweren Kämpfen überhaupt abgerungen worden sind. Die Doppeldeutigkeit, die „funktionale Ambivalenz“ (Blanke) von reformistischen Integrationsprozessen darf also keineswegs vernachlässigt werden. Und diese Ambivalenz gilt sogar in einer Gesellschaft wie der westdeutschen, wo es die ehemalige Arbeiterpartei SPD ist, die nicht nur in den Staat eingetreten ist, sondern seit 1969 im Rahmen der sozialliberalen Koalition diesen Staat ,managt“".

Wir haben schon auf drei Bedingungen aufmerksam gemacht, durch die in der Bundesrepublik die grüne Bewegung ihre Dynamik erlangen konnte: das Anwachsen der ökologischen Probleme, der Verlust politischer Dynamik der Arbeiterbewegung nach dem Kampfzyklus seit Ende der 60er Jahre, die Unfähigkeit der Parteien des herrschenden Blocks, Lösungen für die ökologischen Probleme zu finden. Insofern als traditionell die Sozialdemokratie - auch nach der Wandlung zur „Volkspartei“ - in den lohnabhängigen Massen verankert ist und - dies ist ganz besonders wichtig - von den Gewerkschaften als die Partei betrachtet wird, die am ehesten Interessen der Gewerkschaften in staatliche Politik umzusetzen vermag, kann mit der Kritik an oder gar Gegnerschaft zur Sozialdemokratie und ihrer Politik ein Widerspruch zwischen grïner Bewegung und Arbeiterbewegung schlechthin konstruiert werden und tatsächlich politik relevant werden. Im Prozeß des Regierens verlieren bestimmte Interessen, auch der Arbeiterbewegung, ihre Repräsentanz und werden in den gesellschaftlichen Bereich sozusagen zurückgedrängt, wo sie den Mechanismen bürgerlicher Öffentlichkeit und staatlicher Repression zur Sichenung der Herrschaft (mit der Dynamik, die wir oben angedeutet haben) ausgeliefert werden. Zeitweise gelingt es dadurch der Sozialdemokraiie bzw. der sozialliberalen Koalition gegenüber 
reformistischen Ansprüchen oder grünen Projekten innorhalb des politischen Systems die „Regierbarkeit" des „Modells Deutschland" zu sichern. Aber in der Gesellschaft entsteht auch eine Bewegung, die zunächst nur mit Demonstrationen, Kampagnen, Alternativprojekten usw. von außen das politische System der Institutionen verunsichert, und schließlich den Versuch macht, ihre Interessen auch innerhalb der Institutionen des politischen Systems zur Geltung zu bringen. Darin kommt ein Wandel im Politikverständnis der sozialen Bewegung zum Ausdruck, der nicht zuletzt darauf zurückzuführen ist, daß gerade in der grünen Bewegung nun auch politische Tendenzen zur Protestbewegung stoßen, die eine andere politische Tradition und Kultur repräsentieren als diejenige der Neuen Linken von 1968 oder der neuen sozialen Bewegungen der zweiten Hälfte der 70er Jahre. Konservative und ehemalige Sozialdemokraten, Gewerkschafter und idealistische Humanisten sind nicht von der gleichen Scheu vor dem „Eintritt" in die Institutionen des politischen Systems gezeichnet wie die ,alte“, „neue“ oder ,neue neue" Linke. Also: Dadurch daß der grüne Protest tatsächlich breite Schichten der Bevölkerung erfaßte und von einer anderen als der traditionellen linken Kultur der BRD vorwärtsgetrieben wurde, war die Transfomation des Politikverständnisses und mithin auch die Parteibildung unvermeidlich.

An diesem Punkt ergeben sich innerhalb der grünen Bewegung zwei ganz unterschiedliche Einstellungen. Während die einen in der erfolgten Parteigrïndung einen Verrat gegenüber den Bürgerinitiativen und den anderen Gruppierungen der Ökologiebewegung, gegenüber der Alternativbewegung generell, sehen, begreifen die anderen diese als eine politisch logische Konsequenz der bisherigen Aktivitäten. Bei der Bewertung dieses Widerspruchs sollte allerdings ein entscheidendes Argument nicht vernachlässigt werden: Mit der Parteigründung und ihrer programmatischen Begleitung transformiert sich notwendig die ökologiebewegung in eine politische Organisation, die ihren „Ein-Punkt-Charakter" überwindet. Als Bewegung in der Ge. sellschaft ist die Konzentration auf ein Problem, ein Interesse, einen Punkt möglich; die Organisation dieser Bewegung fungiert letztlich auf der Ebene des politischen Systems als eine Art pressure group, die nicht von Geld, sondern von Masseninteressen getragen wird. Der Hebel an die politische Verwirklichung wird von außen angesetzt. Als Partei agiert die Bewegung in den Institutionen des politischen Systems selbst, wirkt an den En tscheidungen mehr oder weniger subaltern direkt mit und muß daher bereit sein, Kompromisse zu formulieren. Der Rahmen, den die Partei bei den Kompromissen nicht zu verlassen ge denkt, wird als Parteiprogramm - selbst ein Kompromiß zwischen den Strömungen in der Partei - formuliert, das infolgedessen auch für eine Beurteilung der Partei - sofern man es nicht nur als einen Fetzen Papier betrachtet - wichtig wird.

\section{Anmerkungen zum Programm der Grïnen}

Zum Zeitpunkt des Redaktionsschlusses dieses Editorials liegen von Seiten der Grünen ein Programmentwurf und verabschiedete Programmteile vor. Darin wird der 
Transformationsprozeß zu einer ,richtigen“ Partei deutlich. Denn es werden - mit dem Schwerpunkt ökologischer Politik - beinahe alle Punk te einer „Volkspartei“ angesprochen, wobei auch die Alternativforderungen der Gewerkschaftsbewegung beinahe allesamt vertreten sind: Arbeitszeitverkürzungen, Schaffung neuer Arbeitsplätze, Technologie- und Investitionskontrolle, um gesellschaftliche Veränderungen durchzuführen, Kontrolle ökonomischer Macht durch Konzentrationskontrolle, Verbot der Aussperrung und Humanisierung der Arbeitswelt, Umbau der öffentlichen Haushalte im Interesse der Menschen. Bei den Abstimmungen auf dem Saarbrücker Programmparteitag ist großer Wert darauf gelegt worden, gegen den Widerstand von konservativen Grünen Forderungen niederzustimmen, die einen Gegensatz zu den Gewerkschaften konstituieren könnten, und die von den Konservativen ins Auge gefaßsten autoritären Lösungen für die Umweltkrise nicht zum Tragen kommen zu lassen. Grundprinzip ist gewesen: Ökologische Politik und demokratische Bewegung gehören zusammen, ein Widerspruch zur Arbeiterbewegung durch ein wertkonse rvatives Programm muß unbedingt vermieden werden.

Im Programmentwurf kamen diese Tendenzen noch sichtbar zum Ausdruck. Wir wollen auf drei Punkte eingehen, um daran deutlich zu machen, wie ökologische Politik und die sie tragende grüne Bewegung tatsächlich in einen Widerspruch zur Arbeiterbewegung und ihren ökonomischen Interessen geraten können.

1. Der Kritik am ökonomischen (kapitalistischen) Wachstumsmodell entspricht positiv eine Vorstellung von qualitativem Wachstum bzw. Nullwachstum einerseits und einer Dezentralisierung der Produktion zur Herstellung von Überschaubarkeit andererseits. Qualitatives Wachstum wiederum wird wesentlich an der Energieeffektivität der Produktion bemessen, d.h. daran, ob eine Einsparung von Energie und Rohstoffen möglich ist. In diese Richtung zielen denn auch steuerpolitische Änderungen, die jedoch, wenn sie auch Energie und Rohstoffe einsparen mögen, regressive Effekte auf die Einkomme nsverteilung ausüben müssen. Denn anstelle einer Besteuerung der Arbeit durch Lohn- und Einkommensteuer wird für eine Energie- und Rohstoffsteuer plädiert. Wie man aber von allen Verbrauchssteuern weiß, haben diese eine um so größere Bedeutung im Budget der Haushalte, je niedriger das Einkommen ist. Es ist die Frage, ob diese Programmvorstellung nur mangelndem ökonomischem Sachverstand oder aber bewußten politischen Zielsetzungen zuzuschreiben ist. Auch hinsichtlich der Frage der Dezentralisierung bleibt das Programn verschwommen bis problematisch. Auch der Kapitalismus kennt Dezentralisierung der Produktion, etwa in Form ,verlängerter Werkbänke" (d.h. ein Teil der Produktion wird in Länder mit niedrigen Lohnkosten verlagert, sofern die Produktionen arbeitsin tensiv genug sind) oder in Form der in der Region verstreuten Fabrik (fabbrica diffusa), um die Agglomeration von Industriearbeitern unter der Fabrikhalle des Großbetriebs zu zerschlagen, um also die gewerkschaftliche Kampfkraft zu schwächen. Gerade bei der Forderung nach Nullwachstum zeigt sich die schon mehrfach angesprochene Formp roblematik. Denn Nullwachstum an sich führt nicht zu einer Einschränkung von Rohstoff. und Energieverbrauch, sondern nur zu einer Festschreibung des gegebenen Standes des Verbrauchs. Bei Endlichkeit von Energiereserven würde also auch bei Nullwachstum irgendwann, eben nur später, der Punkt er- 
reicht, an dem die Versorgung aufhören würde. Ob unter kapitalistischen Verhältnissen durch Dezentralisierung und kleinbetriebliche Produktionen eine wirkliche Einschränkung des Energieverbrauchs erreicht und die Verwendung alternativer, reproduzierbarer Energiequellen gefördert werden kann, ist mit einem Fragezeichen zu versehen. In Ausfïhrung der beschlossenen Programmteile sind daher noch genauere Überlegungen über die notwendigen Veränderungen industrieller Produktion in ihrer kapitalistischen Form anzustellen, um den ökologischen Ansprüchen und den Interessen der Arbeiterbewegung gerecht zu werden.

2. Auch bei der Forderung nach Verkürzung der Arbeitszeit durch Herabsetzung der flexiblen Altersgrenze, Einführung von Erholungspausen und längerem Urlaub, Verkürzung der wöchentlichen Arbeitszeit, mehr Teilzeitarbeit für Mann und F rau bleibt das Programm einigermaßen widersprüchlich. Während im Entwurf der Programmk ommission noch die Re de davon ist, daß die Arbeitszeit bei vollem Lohnausgleich verkürzt werden soll, treten andere Gruppierungen innerhalb der Grünen auf, die verlangen, die Formulierung ,,bei vollem Lohnausgleich“ im gesamten Text gestrichen wird. An dieser Stelle wird es deutlich, daß innerhalb der Grünen sehr unterschiedliche soziale Interessen und Gruppierungen zu finden sind. Im Programm selbst ist die A rbeitszeitverkürzung bei Lohnausgleich niedergelegt worden.

3. Unter der Überschrift „,Währung“" heißt es im Programmentwurf: ,Die ökologisch-soziale Gleichgewichtswirtschaft führt zu einer stabilen Währung. Die Geldschöpfung ist streng an der Wertschöpfung zu orientieren." Hier haben wir es ganz offensichtlich mit einer monetaristischen Forderung im grünen Gewande zu tun. Denn erstens wird mit einer solchen Formulierung, sollte sie im Programm verankert werden (und sie ist im Programm der Grünen in Baden-Württemberg verankert worden), einer Politik keynesianischen deficit-spending eine klare Absage erteilt worden. Staatliche Politik zur Nachfragestimulie rung, um Arbeitsplätze zu schaffen, wird mit einem solchen Programm verunmöglicht. Und es kommt noch etwas anderes hinzu: Im Monatsbericht der Deutschen Bundesbank vom Januar 1980 wird erstens dargelegt, daß sich die Geldversorgung der Wirtschaft am Produktivitätswachstum zu orientieren habe, um zweitens die Bedingungsfaktoren des Produktivitätswachstums aufzuzeigen. Dabei zeigt es sich dann, daß sich unter kapitalistischen Bedingungen die Produktivität (bzw. um im wertkonservativen Jargon zu sprechen, die Wertschöpfung) gerade auf Kosten der Arbeitskraft erhöhen läßt. Und weiter wird in dem erwähnten Artikel ausgeführt, daß die Verwendung von Kernenergie beispielsweise sehr viel produk tivitätswirksamer sei als die Verwendung herkömmlicher Energiequellen. Die Geldschöpfung an der Wertschöpfung zu orientieren, ist also eine aberwitzige Programmformulierung für eine grüne Partei. Daß es sich hierbei leider nicht nur um eine Programmformulierung handelt, belegt die Politik der Grünen in Bremen im Landesparlament. Wie Rudolf Hickel in einer Analyse grüner Haushaltspolitik in Bremen nachweist (Die Neue vom 11. 3. und 12. 3. 1980), haben sie in Umsetzung dieses Programmsatzes dafür mitgesorgt, daß Streichungen im Sozialetat durchgeführt werden. Hier wird eine Klarstellung absolut notwendig, damit grüne Politik nicht in die Nähe der Biedenkopf und Co. gerät, die mit dem Wort der „Ents taatlichung" eine eindeutige Privatisierungsstrategie fahren, auch eine Stra- 
tegie der Privatisierung des Beschäftigungsrisikos, der gerade mit keynesianischer Politik entgegengewirkt werden könnte. Voraussetzung dafür ist allerdings, daß sich die Wirtschaf tspolitik, die Geldschöpfung, nicht an die ,Wertschöpfung“' binden läßt.

Darüber wird gegenwärtig bei den Grünen noch debattiert und es ist zu hoffen - und hoffentlich zu erwarten - $d a ß$ solche den Widerspruch zu den Interessen der Gewerkschaften, der Lohnabhängigen schlechthin, zementierenden Programmsätze gestrichen werden.

\section{Mögliche Schnittpunkte der Diskussion zwischen ökologie- und Arbeiterbewegung}

Wenn wir für die weitere Diskussion zwischen den Sozialisten und der Ökologiebewegung Schnittpunkte der politischen Perspektiven auffinden wollen, dann können diese auf der Interessenebene an zwei Stellen festgemacht werden:

1. Sowohl eine emanzipatorische Arbeiterbewegung wie auch die Ökologiebewegung setzen an Problemen der Reproduktion des Menschen an; beide Bewegungen sind oder werden deshalb gezwungen, zur kapitalistischen Form der Produktivkraftentwicklung, zur Technologie als Mittel der Profitproduktion, ein kritisches Verhältnis zu entwickeln. Allerdings geschieht dies auf unterschiedlichen Ebenen, die nicht von vornherein in einem unmittelbaren Zusammenhang stehen: Im Produktionsproze $\beta$ ist die angewandte Technik betriebswirtschaftlich definiert, d.h. von dem Interesse des sie einsetzenden Kapitalisten geprägt, die Produktion des relativen Mehrwerts zu steigem (oder die ,Kosten“ zu senken). Und diese Zwecksetzung verwirklicht sich schon in der Konstruktion und Entwicklung te chnologischer Voraussetzungen für den Produktionsprozeß (vgl. dazu den Beitrag von Lutz Hieber in diesem Heft). Geht man daher unter Abstraktion von dieser gesellschaftlich geformten Zwecksetzung der Technik von der fertigen Form aus, dann kann es so scheinen, als of die Technik schlechthin oder ,das Industriesystem "Ursache der negativen Folgen für die Reproduktion des Menschen seien.

Die kapitalistische Zweckbestimmung der angewandten Technik zeigt sich aber gerade in ökonomischen Stagnationsphasen besonders deutlich, wenn neue Technologien als „Rationalisierungsinvestitionen“ in den Produktionsprozeß verstärkt eingeführt werden und dort nicht nur eine Grundlage für die Intensivierung der Arbeit, verstärkten Gesundheitsverschleiß und für Dequalifikationsprozesse der Beschä ftigten abgeben, sondern auch Arbeitsplätze vernichten und die Spaltung der Arbeiter in Beschäftigte und Arbeitslose verstärken. Zugleich sind die Gewerkschaften aber in ihrem Abwehrkampf unmittelbar an der Erhaitung der Arbeitsplätze interessiert; im Rahmen des herrschenden Systems sind sie damit an die Interessen des Kapitals zur Erhöhung der Profitproduktion gebunden und dem Konkurrenzkampf der (nationalen) Kapitale ausgeliefert. Denn legt nicht gerade die Erfahrung mit der kapitalistischen Entwicklung in der Bundesrepublik eine Anpassung an eine Politik der Modernisierung der Volkswirtschaft nahe, durch die Prosperität des Kapitals und besonders die Stellung des westdeutschen Kapitals auf dem Weltmarkt und darüber vermittelt die Sicherheit der Arbeitsplätze gewährleistet werden könnte? Zu- 
mindest entspricht eine solche gewerkschaftliche Politik (die sich voll in Gegensatz $\mathrm{zu}$ den Ökologiebewegungen setzt) auch den vorherrschenden Bewußtseinsformen großer Teile der beschäftigten Arbeiter und ist nicht nur Resultat der Politik der Kooperation der Gewerkschaftsbürokratie mit Kapital und Staat, wie es uns neuere „Verschwörungstheorien“ - z. B. jene in Gestalt des „Atomfilzes“ - weis machen wollen. Während sich aber das Interesse der Beschäftigten an einer Sicherung des Arbeitsplatzes im herrschenden System notwendigerweise mit dem des Kapitals an einer Steigerung der Profitproduktion überschneidet, hat diese Stra tegie zugleich die o. a. einschneidenden Konsequenzen für die individuelle Reproduktion der A rbeitskraft (Arbeitsintensivierung, Gesundheitsverschleiß, Dequalifikation, Entlassungen, die besonders die ,Randbelegschaft" treffen).

Diesem Dilemma (das im Zentrum des in dieser ProKla veröffentlichten Roundtable-Gesprächs steht) können die Gewerkschaften im Rahmen der kapitalistischen Produktionsweise nicht entgehen, wenn sie nicht grundsätzliche Alternativen zu diskutieren beginnen, in denen die kapitalistische Formbestimmtheit der Produktion und Verteilung selbst in Zweifel gezogen werden. Und hier setzt auch die Diskussion um Altemativen der Wirtschaftspolitik in der Bundesrepublik und in anderen Ländern Westeuropas an (vgl. dazu die Artikel in ProKla Nr. 37 und den Artikel von Th. Hahn in diesem Heft, der sich auf die Vorläufer dieser Diskussion in der Weimarer Republik bezieht). Da diese Alternativdiskussion von der allgemeinen Reproduktion des unmi ttelbaren Produzenten ausgeht, sind die Überschneidungen mit Forderungen der Ökologiebewegung unübersehbar. Gerade dieser Zusammenhang und dieser allgemeine Ausgangspunkt scheint uns auf Basis der bislang vorherrschenden nachfrageorientierten (und damit auf Wachstum setzenden) Alternativdiskussion in der Bundesrepublik noch nicht herstellbar gewesen zu sein -- ein Problem, das u. E. auch mit dem krisenanaly tischen Ansatz verbunden ist.

Auch die Ökologiebewegungen haben bislang kaum diesen vermittelnden $\mathrm{Be}$ zug auf die Reproduktion innerhalb und außerhalb der Produktionssphäre thematisiert. Auch wenn dazu Ansätze vorhanden sind, die z, B. die kapitalistischen Prinzipien der Entwicklung und Anwendung der Technologie im Produktionsprozeß kritisch aufgreifen (wie dies im Programmentwurf der Grünen geschieht), kann man doch kaum an diesem Punkt von einem in dieser Richtung verallgemeinerten Selbstverständnis der verschiedenen Ökologiebewegungen reden. Wenn diese Bewegungen aber nicht in die Sackgasse des hilflosen Kampfes gegen die „externen Effekte der Produktion" landen wollen (wo sie sich dann in trauter Gemeinschaft mit der Umweltpolitik der bürgerlichen Regierungen wiederfinden), werden sie auch die gesellschaftlichen Prinzipien der Technologie im kapitalistischen Produktionsprozeß thematisieren müssen. Denn gerade weil die ökologieprobleme, als ,externe Kosten der Produktion", nicht auf einzelne gesellschaftliche Bereiche begrenzbar sind und selbst über die nationalen Grenzen hinausreichen, wird die gesellschaftliche Kontrolle der Produktion eine zentrale Voraussetzung einer veränderten Produktionsweise sein, in der der Gegensatz von Ökologie und Ökonomie aufgehoben, in der die Ökonomie in der Anwendung der Arbeitskraft und des konstanten Kapitals ersetzt werden müßte durch eine Produktion (und das schließt die Produkte ein - vgl. M. Coo- 
leys Beitrag im Roundtable-Gespräch), die dem Menschen dient.

2. Es ist eine alte Erkenntnis, daf die Arbeiterbewegung in Richtung auf die Etablierung einer gesellschaftlich-bewußten Produktionsweise hinarbeiten muß, wenn sie nicht immer wieder durch die naturwüchsigen Resultate der kapitalistischen Produktionsweise zurïkgeworfen werden will (und schließlich haben ja auch die Rückschläge der deutschen Gewerkschaftsbewegung in den 70er Jahren einen Lernprozeß eingeleitet). Eine Erkenntnis allerdings, die gerade nach der Niederlage durch den Faschismus und im Gefolge der Prosperität im Nachkriegsdeutschland weitgehend verschüttet wurde. Damit soll nicht die Schwierigkeit bei der Umsetzung einer solchen Erkenntnis in praktische Politik umgangen werden (vgl. dazu auch unsere Diskussion um die „Krise des Marxismus“ in der ProKla Nr. 36). Diese kann ja nicht nur positiv an die Erfahrung der Ausbeutung im Produktionsprozeß anknüpfen, sondern ist zugleich mit der individuellen Verarbeitung dieser Erfahrung durch den Arbeiter, seiner Disziplinierung im Produktionsprozeß und durch die außen stehende industrielle Reservearmee, und seiner Hoffnung auf die Sicherung des eigenen Arbeitsplatzes durch mehr Leistung oder durch die Erhöhung der Rentabilität des Unternehmens (mehr Wachstum $=$ mehr Arbeitsplätze) konfrontiert.

Die Ökologiebewegung steht demgegenüber heute in einem anderen Gegensatz zur herrschenden Produktionsweise, da ja in der Regel gemeinsame Interessen mit dem Kapital, wie sie oberflächlich die Arbeiter vermittelt über ihr Arbeitsplatzinteresse haben, nicht vorhanden sind (von der Umweltschutzindustrie einmal abgesehen). Während sie einerseits deshalb entschiedener den Kampf für die Erhaltung der natürlichen und gewachsenen Grundlagen des gesellschaftlichen Lebens ansetzen kann, steht sie aber auch andererseits nicht in einem eindeutigen Bezug zur kapitalistischen Produktionsweise, was die Entwicklung z. B. reiner Naturschutzbewegungen erklärt. Dies zeigt sich auch darin, daß oft innerhalb der Ökologiebewegung die kapitalistische Produktionsweise mit dem „Industriesystem“ schlechthin identifiziert wird (darin bestärkt durch die Formen des Umgangs mit der Umwelt in den Staaten des Warschauer Pakts). Eine bewußt-gesellschaftliche Produktinsweise auf Basis der Umwälzung der kapitalistischen Eigentumsverhältnisse als Voraussetzung einer ökologisch verantworungsbewußten Produktionsweise wird deshalb auch innerhalb der Ökologiebewegung (noch) nicht allgemein diskutiert und daran ist $u$. E. auch die schlech te Tradition der Zweiten und Dritten Internationalen (die sich heute gleichermaßen in SPD wie DKP fortsetzt) nicht unschuldig. Schließlich konnte diese nur in der rücksichtslosen Produktivkraftentwicklung ihr Heil sehen und die gesellschaftlichen Konsequenzen der ,objektiv notwendigen“ Umwälzung nur in der Zentralisierung und Verstaatlichung sehen (am Beispiel der Diskussion um die Produktivkraftentwicklung in der Weimarer Republik bei SPD, KPD und ADGB in ProKla Nr. 31 und am Beispiel des Sozialstaats in diesem Heft hat Chr. Neusüß diese Problematik aufgegriffen und diskutiert). Dagegen setzt die Ökologiebewegung eher die gesellschaftliche Dezentralisierung, die kleinen menschengerechteren Einheiten und die alternative Technologie - aber in welcher gesellschaftlichen Form? Wie soll sich der Zusammenhang zwischen diesen Einheiten im Inland und im in ternationalen Zusammenhang konstituieren, wenn nicht auf eine konkur- 
renzhafte Form zurückgegriffen werden soll (die Marx ja schon in seiner Polemik gegen Proudhon zu Recht radikal kritisierte)? An diesem Punkt dürfte auch die Ökologiebewegung früher oder später auf die Frage einer gesellschaftlich notwendigen Umwälzung der Produktionsweise gelangen und dann auch die Vorstellungen einer gesellschaftlichen Veränderung von Teilen der Arbeiterbewegung mitdiskutieren müssen. (Sowohl gesamtgesellschaftliche Überlegungen im Rahmen des BBU als auch die Programmdiskussion in Saarbrücken bei den Grünen haben diese Probleme auch aufgegriffen.)

Arbeiter- und Ökologiebewegung - ein Widerspruch? Allerdings, und dieser Widerspruch muß auch ausgetragen werden. Nur: einen unvermittelbaren Gegensatz konstituieren diese beiden Bewegungen nicht.

Der zu diesem Heft mehrfach angekündigte Artikel von Wolfgang Schöller über die Anwendung moderner Technologie in den En twicklungsländern und ihren Folgen muß auf ein späteres Hef $t$ verschoben werden. Die nächsten beiden Hefte der ProKla werden dem ,Modell Deutschland" gewidmet sein: Heft 40 soll zunächst Analy sen und Diskussionen von bundesdeutschen Autoren enthalten, in Heft 41 sollen dann Einschätzungen ausländischer Autoren veröffentlicht werden. 